\title{
Mulheres e a questão racial da sífilis no Brasil: uma análise de tendência (2010-2019)
}

\author{
Women and the racial issue of syphilis in Brazil: a trend analysis (2010-2019) \\ Mujeres y la cuestión racial de la sífilis en Brasil: un análisis de tendencia (2010-2019)
}

Recebido: 28/12/2021 | Revisado: 03/01/2022 | Aceito: 11/01/2022| Publicado: 13/01/2022

\author{
Michael Ferreira Machado \\ ORCID: https://orcid.org/0000-0001-6538-6408 \\ Universidade Federal de Alagoas, Brasil \\ E-mail: michael.machado@ arapiraca.ufal.br \\ Bruna Stefany Rebouças França \\ ORCID: https://orcid.org/0000-0002-7730-9562 \\ Universidade Federal de Alagoas, Brasil \\ E-mail: bruna.franca@ arapiraca.ufal.br \\ Maria Lua Santos Alves de Farias \\ ORCID: https://orcid.org/0000-0002-1807-9533 \\ Universidade Federal de Alagoas, Brasil \\ E-mail: maria.lua@arapiraca.ufal.br \\ Mariana Ivo Costa \\ ORCID: https://orcid.org/0000-0003-0784-1163 \\ Universidade Federal de Alagoas, Brasil \\ E-mail: mariana.ivo@ arapiraca.ufal.br
}

\begin{abstract}
Resumo
Este estudo objetivou analisar a relação entre a ocorrência de sífilis e a população feminina negra nas cinco regiões geográficas brasileiras e no Brasil entre 2010-2019. Trata-se de um estudo observacional ecológico que utilizou dados secundários disponibilizados pelo Instituto Brasileiro de Geografia e Estatística e pelo Departamento de Doenças Crônicas e Infecções Sexualmente Transmissíveis do Ministério da Saúde. No tratamento estatístico dos dados, foi utilizado o software Jointpoint Regression Program, versão 3.3.1, para analisar as tendências no recorte temporal estabelecido. Os resultados apontam que, apesar de as mulheres pretas e pardas representarem quase metade da população feminina brasileira, estas não possuem o mesmo acesso à saúde que mulheres brancas. Tal fato gera iniquidades que, associadas à discriminação racial, trazem consequências permanentes para saúde de indivíduos e coletividades; e contribuem para a adoção de comportamentos inadequados e para o adoecimento, aumentando a possibilidade de ocorrência de casos de doenças infecciosas, como a sífilis. A essa problemática, soma-se inconformidades nos registros sobre autodeclaração racial de saúde. Assim, o adequado preenchimento das fichas de notificação, bem como a elaboração e execução de políticas públicas voltadas à geração de equidade na saúde podem contribuir para atenuação e enfrentamento da sífilis na população negra.
\end{abstract}

Palavras-chave: Sífilis; Racismo; Saúde da mulher; Vulnerabilidade social; Saúde pública.

\begin{abstract}
This study aimed to analyze the relationship between the occurrence of syphilis and the black female population in the five Brazilian geographic regions and in Brazil between 2010-2019. This is an ecological observational study that used secondary data provided by the Brazilian Institute of Geography and Statistics and by the Department of Chronic Diseases and Sexually Transmitted Infections of the Ministry of Health. In the statistical treatment of the data, was used the software Jointpoint Regression Program version 3.3.1, to analyze trends in the established time frame. The results show that, although black and brown women represent almost half of the Brazilian female population, they do not have the same access to health care as white women. This fact generates inequities that, associated with racial discrimination, bring permanent consequences for the health of individuals and communities; and contribute to the adoption of inappropriate behaviors and to illness, increasing the possibility of occurrence of cases of infectious diseases, such as syphilis. In addition to this problem, there are non-conformities in the records on racial self-declaration of health. Thus, the proper completion of notification forms, as well as the elaboration and execution of public policies aimed at generating equity in health can contribute to the attenuation and confrontation of syphilis in the black population.
\end{abstract}

Keywords: Syphilis; Racism; Women's health; Social vulnerability; Public health.

\section{Resumen}

Este estudio tuvo como objetivo analizar la relación entre la ocurrencia de sífilis y la población femenina negra en las cinco regiones geográficas brasileñas y en Brasil entre 2010-2019. Se trata de un estudio observacional ecológico que utilizó datos secundarios proporcionados por el Instituto Brasileño de Geografía y Estadística y por el Departamento de 
Enfermedades Crónicas e Infecciones de Transmisión Sexual del Ministerio de Salud. En el tratamiento estadístico de los datos, se utilizó el software Jointpoint Regression Program, versión 3.3.1, para analizar las tendencias en el período de tiempo establecido. Los resultados apuntan que, aunque las mujeres negras representan casi la mitad de la población femenina brasileña, no poseen el mismo acceso a la salud que las mujeres blancas. Este hecho genera inequidades que, asociadas a la discriminación racial, traen consecuencias permanentes para la salud de individuos y colectividades; y contribuyen para la adopción de conductas inadecuadas y para la enfermedad, aumentando la posibilidad de ocurrencia de casos de enfermedades infecciosas, como la sífilis. A esta problemática se suma inconformidades en los registros de autodeclaración racial de salud. Así, la adecuada cumplimentación de las fichas de notificación, así como la elaboración y ejecución de políticas públicas dirigidas a la generación de equidad en salud pueden contribuir para la atenuación y enfrentamiento de la sífilis en la población negra.

Palabras clave: Sífilis; Racismo; Salud de la mujer; Vulnerabilidad social; Salud pública.

\section{Introdução}

A história do Brasil foi escrita, por mais de três séculos, sob a égide da escravidão. Nesse sistema, inúmeros indivíduos foram trazidos do continente africano e forçadamente inseridos como mão de obra nos ciclos econômicos vigentes nos períodos colonial e imperial. Apesar de o comércio transatlântico de produtos e seres humanos ter sido uma atividade lucrativa, o quadro social gerado pela escravidão produziu sequelas que, ainda hoje, se perpetuam no cotidiano brasileiro (Mariosa, 2019).

O negro escravizado - vítima evidente de todo o processo de colonização - era visto apenas como um item econômico e, portanto, desprovido de direitos dos quais os brancos podiam se beneficiar e privado até mesmo de participar das formas de vida social organizadas mínimas, como a família (Mariosa, 2019). Esse processo de desumanização do negro africano traficado ao Brasil e, posteriormente, dos negros nascidos dentro dos limites do território brasileiro, foi resultado de uma ideologia que colocava o branco europeu em patamar de superioridade em relação aos povos dominados. Ao atribuir aos escravizados a condição de subalterno, verticalizando a relação, tentava-se justificar o próprio processo escravocrata e legitimar todas as violências produzidas a partir dele: o açoite, a flagelação, a mutilação e o domínio sobre os corpos negros (Santos, 2015).

Apesar de ter garantido legalmente a liberdade, a abolição da escravidão, ocorrida em maio de 1888, não forneceu a base social necessária à integração do cidadão negro na sociedade brasileira, pois esta continuava sujeita aos interesses políticos, culturais e econômicos e reproduzia a ideologia dos estratos superiores (Mariosa, 2019).

No período pós-abolição, a população negra foi marginalizada e relegada aos ambientes mais inóspitos das cidades brasileiras; passando a viver em áreas de risco e nas periferias, posteriormente conhecidas como favelas. Não houve, por parte do governo brasileiro, interesse em garantir o acesso dessa população aos direitos fundamentais, como educação, moradia, infraestrutura e saúde. A ausência de políticas públicas voltadas a essa parcela da população acentuou as desigualdades sociais e culminou no que, atualmente, conhece-se como racismo institucional (da Cruz Santos, 2019), refletido, até hoje, nos indicadores sociais.

O racismo é um fenômeno ideológico, que se constitui como agente de violação de direitos e de produção de iniquidades, inclusive no campo da saúde. Ele exerce impacto sobre a qualidade do cuidado e da assistência prestados, sobre os índices de mortalidade materna e infantil e relaciona-se, ainda, com os desfechos desfavoráveis que poderiam ser evitados (Batista et al., 2013). Apesar de a Constituição Federal de 1988 assegurar, em seu artigo 196, o acesso universal à saúde, para Lopes os serviços de saúde não têm oferecido aos negros o mesmo nível, qualidade de atenção e perfil de saúde apresentado pelos brancos. Negros e brancos ocupam lugares diferentes e carregam experiências também desiguais ao nascer, viver, adoecer e morrer (Lopes, 2005).

Nessa perspectiva, é importante entender que no âmbito da saúde, o racismo institucional se apresenta por meio de iniquidades sofridas por mulheres e homens negros, de todas as regiões do país, ao longo da vida, sendo esta condição uma das causas da hierarquização social que se associa a vulnerabilidades em saúde reveladas pelo agravamento das condições de saúde e taxas de mortalidade em negros quando comparadas às de outros agrupamentos étnico-raciais (Werneck, 2016).

A situação agrava-se na mulher negra, pois soma-se ao racismo outros elementos, como o machismo, e preconceitos 
que lhe são destinados e variam conforme a religião seguida, a orientação sexual e o poder socioeconômico (de Paiva Terra, 2020). Dessa forma, há uma interseccionalidade entre diversas e simultâneas opressões historicamente conhecidas sobre essas mulheres (Crenshaw, 1989). As mulheres negras estão na base da pirâmide da valorização social, em situação de maior vulnerabilidade à exposição às infecções sexualmente transmissíveis (IST), com menor autonomia sobre o próprio corpo e menor acesso à saúde (Santos, 2016; Souzas, et al., 2012).

Dentre essas infecções, a sífilis ganha destaque no cenário nacional, pois acomete parte expressiva da população brasileira, inclusive a mulher negra. Essa doença apresenta como agente etiológico a bactéria Treponema pallidum e pode se apresentar clinicamente nas formas adquirida, gestacional ou congênita. Também possui diferentes estágios, sendo eles primário, secundário, latente e terciário, expressando o último maiores sinais de gravidade (Souzas et al., 2012).

Por conseguinte, o objetivo deste estudo é analisar a relação entre a ocorrência de sífilis e a população feminina negra nas diferentes regiões geográficas brasileiras e no Brasil entre 2010-2019.

\section{Metodologia}

Trata-se de um estudo observacional ecológico (Almeida \& Barreto, 2011) que utiliza dados socioeconômicos disponibilizados pelo Instituto Brasileiro de Geografia e Estatística e dados secundários disponibilizados pelo Departamento de Doenças Crônicas e Infecções Sexualmente Transmissíveis do Ministério da Saúde para analisar as taxas de sífilis adquirida, gestacional e congênita em mulheres negras no período entre 2010 a 2019. Os dados utilizados no estudo foram colhidos no dia 31 de março de 2021.

As informações coletadas foram tabuladas em planilhas eletrônicas do programa Microsoft Excel 2016. Posteriormente, utilizou-se o software Jointpoint Regression Program, versão 3.3.1 (Statistical Research and Applications Branch, National Cancer Institute, Bethesda, Estados Unidos. http://srab.cancer.gov/joinpoint) para analisar as tendências temporais dos coeficientes de sífilis adquirida, sífilis gestacional e sífilis congênita em mulheres negras, avaliando se há alterações do padrão de tendência observado (crescente ou decrescente) ou não (estacionário).

O Jointpoint utiliza a variável dependente em uma escala logarítmica para aplicar variados testes de permutação de Monte Carlo para comparar diversos modelos e encontrar o melhor para representar a tendência temporal. A partir da elucidação do modelo, define-se as Variações Percentuais Anuais (APC - Annual Percentage Change) e Variação Percentual Anual Média (AAPC - Average Annual Percentage Change) para avaliar se a tendência é estatisticamente significativa, considerando os intervalos de $95 \%$ de confiança (IC95\%).

Em conformidade com as resoluções que regulamentam a pesquisa científica no Brasil, o estudo não requereu submissão no Comitê de Ética em Pesquisa (CEP), pois os dados secundários utilizados são de domínio público e disponibilizados pelo Ministério da Saúde e Instituto Brasileiro de Geografia Estatística (IBGE).

\section{Resultados}

A Figura 1 apresenta a distribuição das mulheres pretas e pardas no Brasil. 
Figura 1. Distribuição das mulheres pardas e pretas no Brasil.

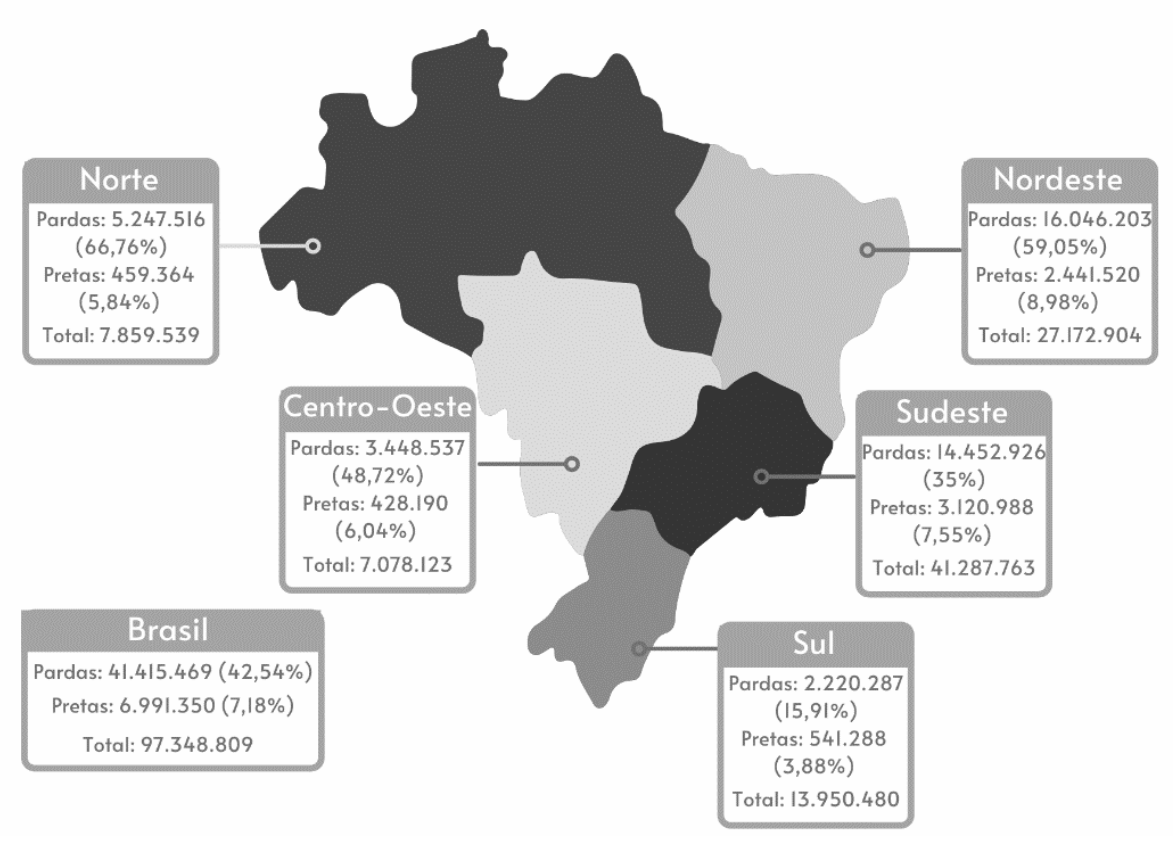

Fonte: Censo Demográfico 2010 - Instituto Brasileiro de Geografia e Estatística (IBGE).

Nele, observa-se que mulheres negras correspondem a 49,72\% do total da população feminina no país. Acima da média nacional, destacam-se a região Norte, com um percentual de 72,6\% de mulheres negras em relação ao total de mulheres nortistas, seguida pelo Nordeste $(68,03 \%)$ e, por fim, pela região Centro-Oeste, que possui mais da metade $(54,76 \%)$ das mulheres pertencentes a esse estrato racial. A distribuição geográfica por raça/cor revela ainda o Sul do Brasil como a região detentora da menor proporção de pardas e pretas dentre todas as outras localidades do país $(19,79 \%)$. No panorama geral, as sulistas representam apenas $5,7 \%$ das mulheres negras brasileiras.

Em relação à ocorrência de sífilis adquirida nas mulheres, demonstrada na Tabela 1, a tendência brasileira mostrou-se crescente (AAPC:0,6; $\mathrm{p}=0,0$ ) entre os anos de 2010 a 2019. Resultados similares foram achados nas regiões Sudeste (AAPC:0,9; $\mathrm{p}=0,0$ ), Centro-Oeste (AAPC:18,5;p=0,0) e Sul (AAPC:27,7; $\mathrm{p}=0,0$ ). 
Tabela 1. Tendências da sífilis adquirida por sexo e ano de diagnóstico no Brasil e em suas regiões geográficas (2010-2019)

\begin{tabular}{|c|c|c|c|}
\hline Período & APC (IC95\%) & AAPC (IC95\%) & Tendência \\
\hline \multicolumn{4}{|c|}{ Brasil } \\
\hline 2010-2019 & $\begin{array}{c}0,6(0,2 \text { a } 1,0) \\
p=0,0\end{array}$ & $\begin{array}{c}0,6(0,2 \text { a } 1,0) \\
p=0,0\end{array}$ & Crescente \\
\hline \multicolumn{4}{|c|}{ Norte } \\
\hline $\begin{array}{l}2010-2013 \\
2013-2019\end{array}$ & $\begin{array}{c}1,8(-2,5 \text { a } 6,3) \\
p=0,3 \\
-2,3(-3,5 \text { a }-1,1) \\
p=0,0\end{array}$ & $\begin{array}{c}-1,0(-2,2 \text { a } 0,3) \\
p=0,1\end{array}$ & Estacionária \\
\hline \multicolumn{4}{|c|}{ Nordeste } \\
\hline $\begin{array}{r}2010-2015 \\
2015-2019 \\
\end{array}$ & $\begin{array}{c}3,5(1,4 \text { a } 5,7) p=0,0 \\
-8,8(-11,6 \text { a }-5,9) p=0,0\end{array}$ & $\begin{array}{l}-2,2(-3,5 a \\
-0,8) p=0,0\end{array}$ & Decrescente \\
\hline \multicolumn{4}{|c|}{ Sudeste } \\
\hline 2010-2019 & $\begin{array}{c}0,9(0,2 \text { a } 1,6) \\
p=0,0\end{array}$ & $\begin{array}{c}0,9(0,2 \text { a } 1,6) \\
p=0,0\end{array}$ & Crescente \\
\hline \multicolumn{4}{|c|}{ Centro-Oeste } \\
\hline 2010-2019 & $\begin{array}{c}18,5(16,6 \text { a } 20,5) \\
p=0,0\end{array}$ & $\begin{array}{c}18,5(16,6 \text { a } 20,5) \\
p=0,0\end{array}$ & Crescente \\
\hline \multicolumn{4}{|c|}{ Sul } \\
\hline $\begin{array}{r}2010-2016 \\
2016-2019\end{array}$ & $\begin{array}{c}37,8(35,4 \text { a } 40,2) \\
p=0,0 \\
9,6(2,8-16,8) \\
p=0,0\end{array}$ & $\begin{array}{c}27,7(25,3 \text { a } 30,0) \\
p=0,0\end{array}$ & Crescente \\
\hline
\end{tabular}

Fonte: MS/SVS/Departamento de Doenças Crônicas e Infecções Sexualmente Transmissíveis

Entretanto, a tendência da sífilis adquirida em mulheres apresentou-se decrescente na região Nordeste (AAPC:-2,2, $\mathrm{p}=0,0$ ) e permaneceu estacionária no Norte do país (AAPC:-1,0; $\mathrm{p}=0,1$ ).

De acordo com a Tabela 2, entre os anos de 2010 a 2019, a taxa de detecção da sífilis gestacional apresentou-se crescente no Brasil (AAPC:23,3; p=0,0), tendência similar às encontradas nas regiões Norte (AAPC:19,8; p=0,0), Nordeste (AAPC:22,0; p=0,0) e Sudeste (AAPC: 23,3; p=0,0); e oposta à encontrada no Centro-Oeste (AAPC:-2,3; p=0,0). No Sul, a taxa de detecção da sífilis gestacional mostrou-se estacionária durante todo o período analisado (AAPC: 0,6; p=0,4). 
Tabela 2. Taxa de detecção e tendências da sífilis gestacional no Brasil e em suas regiões geográficas (2010-2019).

\begin{tabular}{|c|c|c|c|c|}
\hline Característica & Período & APC (IC95\%) & AAPC (IC95\%) & Tendência \\
\hline \multicolumn{5}{|c|}{ Brasil } \\
\hline Taxa de Detecção SG & $2010-2019$ & $23,3(21,7$ a 24,9$) \mathrm{p}=0,0$ & $23,3(21,7$ a 24,9$) \mathrm{p}=0,0$ & Crescente \\
\hline SG: Pretas & $2010-2019$ & $-0,3(-1,0$ a 0,3$) p=0,2$ & $-0,3(-1,0$ a 0,3$) p=0,2$ & Estacionária \\
\hline SG: Pardas & $\begin{array}{l}2010-2016 \\
2016-2019 \\
\end{array}$ & $\begin{array}{l}0,2(-0,1 \text { a } 0,5) p=0,1 \\
3,3(2,2 \text { a 4,4) } p=0,0\end{array}$ & $1,2(0,9$ a 1,6$) p=0,0$ & Crescente \\
\hline \multicolumn{5}{|c|}{ Norte } \\
\hline Taxa de Detecção SG & $2010-2019$ & $19,8(16,6$ a 23,1$) \mathrm{p}=0,0$ & $19,8(16,6$ a 23,1$) p=0,0$ & Crescente \\
\hline SG: Pretas & $2010-2019$ & $-3,5(-4,9$ a $-2,0) p=0,0$ & $-3,5(-4,9 a-2,0) p=0,0$ & Decrescente \\
\hline SG: Pardas & $2010-2019$ & $0,2(-0,1$ a 0,6$) p=0,1$ & $0,2(-0,1$ a 0,6$) p=0,1$ & Estacionária \\
\hline \multicolumn{5}{|c|}{ Nordeste } \\
\hline Taxa de Detecção SG & $2010-2019$ & $22(19,5$ a 24,7$) \mathrm{p}=0,0$ & $22(19,5$ a 24,7$) \mathrm{p}=0,0$ & Crescente \\
\hline SG: Pretas & $\begin{array}{l}2010-2017 \\
2017-2019\end{array}$ & $\begin{array}{c}1,9(-2,2 \text { a } 6,2) p=0,3 \\
-17,2(-41,4 \text { a } 17,2) \\
p=0,2\end{array}$ & $-2,7(-8,7$ a 3,7$) \mathrm{p}=0,4$ & Estacionária \\
\hline SG: Pardas & $2010-2019$ & $1,2(0,8$ a 1,6$) \mathrm{p}=0,0$ & $1,2(0,8$ a 1,6$) p=0,0$ & Crescente \\
\hline \multicolumn{5}{|c|}{ Sudeste } \\
\hline Taxa de Detecção SG & $2010-2019$ & $23,3(20,5$ a 26,1$) p=0,0$ & $23,3(20,5$ a 26,1$) p=0,0$ & Crescente \\
\hline SG: Pretas & $2010-2019$ & $0,5(-0,2$ a 1,2$) p=0,1$ & $0,5(-0,2$ a 1,2$) p=0,1$ & Estacionária \\
\hline SG: Pardas & $\begin{array}{l}2010-2017 \\
2017-2019 \\
\end{array}$ & $\begin{array}{c}3(2,7 \text { a } 3,2) \\
p=0,0 \\
1,3(-1,2 \text { a } 3,9) p=0,2\end{array}$ & $2,6(2,1$ a 3,1$) p=0,0$ & Crescente \\
\hline \multicolumn{5}{|c|}{ Centro-Oeste } \\
\hline Taxa de Detecção SG & $2010-2019$ & $-2,3(-3,6 a-1,1) p=0,0$ & $-2,3(-3,6 a-1,1) p=0,0$ & Decrescente \\
\hline SG: Pretas & $2010-2019$ & $-1,0(-3,3$ a 1,3$) p=0,3$ & $-1,0(-3,3$ a 1,3$) p=0,3$ & Estacionária \\
\hline SG: Pardas & $2010-2019$ & $3,1(2,6$ a 3,5$) p=0,0$ & $3,1(2,6$ a 3,5$) p=0,0$ & Crescente \\
\hline \multicolumn{5}{|c|}{ Sul } \\
\hline Taxa de Detecção SG & $\begin{array}{l}2010-2012 \\
2012-2019 \\
\end{array}$ & $\begin{array}{c}-3,1(-10,2 \text { a } 4,4) p=0,3 \\
1,6(1,0 \text { a } 2,3) p=0,0\end{array}$ & $0,6(-0,8$ a 1,9$) \mathrm{p}=0,4$ & Estacionária \\
\hline SG: Pretas & $2010-2019$ & $-2,0(-3,3$ a $-0,6) p=0,0$ & $-2,0(-3,3$ a $-0,6) p=0,0$ & Decrescente \\
\hline SG: Pardas & $2010-2019$ & $0,9(-0,0$ a 1,9$) p=0,1$ & $0,9(-0,0$ a 1,9$) p=0,1$ & Estacionária \\
\hline
\end{tabular}

Fonte: MS/SVS/Departamento de Doenças Crônicas e Infecções Sexualmente Transmissíveis

Quanto à ocorrência da sífilis gestacional, não foram observadas tendências crescentes em nenhuma região brasileira. Todas as regiões apresentaram-se estacionárias para esse quesito, com exceção do Sul do Brasil, na qual a disposição encontrada mostrou-se decrescente (AAPC:-2,0; $\mathrm{p}=0,00$ ). Para as pardas, entretanto, as tendências apresentaram-se crescentes no Nordeste (AAPC: 1,2; $\mathrm{p}=0,0$ ), Sudeste (AAPC: 2,6; $\mathrm{p}=0,0$ ) e no Centro-Oeste (AAPC: 3,1; $\mathrm{p}=0,0$ ); sendo similares à tendência de sífilis gestacional nacional (AAPC:1,2; p=0,0). Nas regiões Norte e Sul, os resultados encontrados foram de tendências estacionárias.

Os dados presentes na Tabela 3 revelam que, entre os anos de 2010 a 2019, não houve presença de tendências crescentes referentes à ocorrência de sífilis congênita em filhos de mulheres pretas. Os resultados encontrados foram decrescentes nas regiões Norte (AAPC:-7,9; $\mathrm{p}=0,0$ ), Sudeste (AAPC:-3,2; $\mathrm{p}=0,0$ ) e Sul (AAPC:-2,0; $\mathrm{p}=0,0$ ), semelhantemente ao ocorrido com a tendência nacional (AAPC:-2,9; $\mathrm{p}=0,0$ ). No Nordeste e no Centro-Oeste, os achados mostraram-se estacionários. 
Tabela 3. Tendências da sífilis congênita em mulheres pretas e pardas no Brasil e em suas regiões geográficas (2010-2019)

\begin{tabular}{|c|c|c|c|c|}
\hline Característica & Período & APC (IC95\%) & AAPC (IC95\%) & Tendência \\
\hline \multicolumn{5}{|c|}{ Brasil } \\
\hline SC: Pretas & $2010-2019$ & $-2,9(-3,5$ a 2,2$) p=0,0$ & $-2,9(-3,5$ a 2,2$) \mathrm{p}=0,0$ & Decrescente \\
\hline SC: Pardas & $2010-2019$ & $1,0(0,7$ a 1,4$) p=0,0$ & $1,0(0,7$ a 1,4$) p=0,0$ & Crescente \\
\hline \multicolumn{5}{|c|}{ Norte } \\
\hline SC: Pretas & $2010-2019$ & $-7,9(-9,1$ a $-6,7) p=0,0$ & $-7,9(-9,1 \mathrm{a}-6,7) \mathrm{p}=0,0$ & Decrescente \\
\hline SC: Pardas & $2010-2019$ & $1,2(1,0$ a 1,4$) p=0,0$ & $1,2(1,0$ a 1,4$) p=0,0$ & Crescente \\
\hline \multicolumn{5}{|c|}{ Nordeste } \\
\hline SC: Pretas & $\begin{array}{l}2010-2017 \\
2017-2019\end{array}$ & $\begin{array}{c}1,0(-1,3 \text { a } 3,4) \mathrm{p}=0,3 \\
-11,7(-37,2 \text { a } 24,1) \\
\mathrm{p}=0,4\end{array}$ & $-2,0(-7,6$ a 4,0$) \mathrm{p}=0,5$ & Estacionária \\
\hline SC: Pardas & $2010-2019$ & $0,7(0,3$ a 1,1$) \mathrm{p}=0,0$ & $0,7(0,3$ a 1,1$) \mathrm{p}=0,0$ & Crescente \\
\hline \multicolumn{5}{|c|}{ Sudeste } \\
\hline SC: Pretas & $2010-2019$ & $-3,2(-4,1$ a $-2,2) p=0,0$ & $-3,2(-4,1 \mathrm{a}-2,2) \mathrm{p}=0,0$ & Decrescente \\
\hline SC: Pardas & $2010-2019$ & $3,4(2,9$ a 3,9$) \mathrm{p}=0,0$ & $3,4(2,9$ a 3,9$) \mathrm{p}=0,0$ & Crescente \\
\hline \multicolumn{5}{|c|}{ Centro-Oeste } \\
\hline SC: Pretas & $\begin{array}{l}2010-2016 \\
2016-2019\end{array}$ & $\begin{array}{l}-6,4(-12,4 \mathrm{a}-0,1) \mathrm{p}=0,0 \\
18,9(-7,2-52,3) \mathrm{p}=0,1\end{array}$ & $1,3(-5,6$ a 8,8$) p=0,7$ & Estacionária \\
\hline SC: Pardas & $2010-2019$ & $0,8(-0,1$ a 1,8$) p=0,1$ & $0,8(-0,1$ a 1,8$) p=0,1$ & Estacionária \\
\hline \multicolumn{5}{|c|}{ Sul } \\
\hline SC: Pretas & $2010-2019$ & $-2,0(-3,3$ a $-0,6) p=0,0$ & $-2,0(-3,3$ a $-0,6) p=0,0$ & Decrescente \\
\hline SC: Pardas & $2010-2019$ & $1,3(-0,3$ a 2,9$) p=0,1$ & $1,3(-0,3$ a 2,9$) p=0,1$ & Estacionária \\
\hline
\end{tabular}

Fonte: MS/SVS/Departamento de Doenças Crônicas e Infecções Sexualmente Transmissíveis

Para filhos de mulheres pardas, as tendências apresentaram-se estacionárias no Centro-Oeste e no Sul; e crescentes nas regiões Norte (AAPC:1,2; $=0,0$ ), Nordeste (AAPC:0,7; $=0,0$ ) e Sudeste (AAPC:3,4; $\mathrm{p}=0,0$ ), assim como a tendência nacional (AAPC: 1,$0 ; \mathrm{p}=0,0)$.

Portanto, não foram encontradas tendências decrescentes na ocorrência de sífilis congênita em mulheres pretas e pardas em nenhuma das regiões brasileiras, assim como no Brasil, entre o período de 2010 e 2019.

\section{Discussão}

Ao pesquisar a tendência da sífilis em mulheres pretas e pardas em todas as regiões brasileiras, assim como no Brasil, pode-se dimensionar um dos efeitos do racismo histórico e institucional na vida dessas mulheres, revelando que a sífilis, enquanto problema de saúde pública, apresenta agravos sob a perspectiva de raça. De maneira geral, observa-se que mulheres negras apresentam tendências decrescentes ou estacionárias no que se diz respeito à detecção de sífilis, seja adquirida, gestacional ou congênita.

Essas mulheres, apesar de representarem metade da população feminina brasileira $(49,72 \%)$, não possuem o mesmo acesso ao sistema de saúde que mulheres brancas, pois o alcance a recursos e dispositivos necessários para os cuidados em saúde são inferiores aos dispostos para estas. Corroborando com essa afirmação, em estudos desenvolvidos sobre o tema, identificouse um gradiente de cuidado do menos satisfatório para mais satisfatório entre pretas, pardas e brancas que resultam em benefícios e oportunidades diferenciadas segundo raça/cor, com prejuízo para as mulheres de pele retinta (Brasil, n.d,; Leal et al., 2017).

Especialmente no âmbito da saúde materno-infantil, essa desigualdade pode ser observada a partir do cenário epidemiológico da sífilis no Brasil. As manifestações clínicas dessa infecção sexualmente transmissível são especialmente graves no período gestacional ou quando ocorre transmissão congênita, quando a gestante com sífilis não é tratada ou é tratada de forma inadequada, resultando na passagem do Treponema pallidum para o feto por via hematogênica (Avelleira \& Bottino, 
2006). A sífilis apresenta-se em variados estágios: primário, secundário, latente e terciário, sendo que nos dois primeiros ocorre maior possibilidade de transmissão da bactéria ao concepto (Brasil, n.d.). Trata-se, portanto, de um grave problema de saúde pública que evidencia as desigualdades relativas ao acesso à saúde entre os diferentes agrupamentos étnico-raciais presentes no Brasil.

A ocorrência de sífilis adquirida, crescente no Brasil nos anos analisados, está associada a situações de vulnerabilidades. Segundo as publicações oficiais do Ministério da Saúde, apesar da pequena redução no percentual de casos em indivíduos analfabetos ou com ensino fundamental incompleto, além de um aumento no percentual de casos em indivíduos com ensino fundamental ou médio completo no recorte temporal de 2010 a 2019, maiores índices da doença ainda são encontrados em pessoas negras (48,3\% das notificações) (Brasil, 2020), de estratos sociais mais desfavorecidos, com menores níveis de escolaridade e renda e acesso limitado a informações e insumos como preservativos (Macêdo et al., 2017).

Como reflexo dessas vulnerabilidades, documentos produzidos pelo Instituto Brasileiro de Geografia e Estatística (IBGE), revelaram que, no ano de 2019, entre as mulheres negras na faixa etária de 15 a 29 anos, $32 \%$ não estavam estudando e não possuíam ocupação. Para jovens brancos na mesma situação, a taxa foi de $13,2 \%$, proporção 2,4 vezes menor que a apresentada para os negros. A população feminina negra também se evidenciou entre os pobres, representando $28,7 \%$ da população brasileira e 39,8\% dos extremamente pobres. O arranjo domiciliar formado por mulheres pretas ou pardas responsáveis pela subsistência familiar, sem cônjuge e com presença de filhos menores de 14 anos concentrou a maior incidência de pobreza: $24 \%$ desses moradores apresentavam rendimento domiciliar per capita menor que US\$1,90 e 62,4\% inferior a US\$ 5,50 (Instituto Brasileiro de Geografia e Estatística [IBGE], 2019).

Entretanto, em anos anteriores, os dados socioeconômicos disponíveis em documento do Ministério da Saúde, publicado em 2005, já indicavam que a maioria das mulheres negras encontrava-se abaixo da linha da pobreza, representando a seguinte situação: taxa de analfabetismo era o dobro das mulheres brancas; eram, em maioria, chefes de família, sem cônjuge e com filhos; e, por fatores sociais e discriminatórios, obtinham menor acesso aos serviços de saúde de qualidade e à assistência ginecoobstétrica; além do maior risco, em comparação a mulheres brancas, de adquirir e ir a óbito mais cedo por determinadas doenças (IBGE, 2019).

Nas regiões Centro-Oeste, Sudeste e Sul, as tendências crescentes para a sífilis adquirida, semelhantes à tendência nacional, podem ser explicadas pela dificuldade na captação de mulheres com vida sexual ativa para estabelecimento do diagnóstico precoce, que pode ser obtido a partir da combinação de dados clínicos associados a testes diagnósticos, histórico de infecções anteriores e investigação de recente exposição sexual de risco, assim como o seguimento com tratamento imediato com penicilina benzatina, após um teste reagente para sífilis, mesmo sem a presença de sinais e sintomas. Segundo preconiza o Protocolo Brasileiro para Infecções Sexualmente Transmissíveis (2020), para superar esse agravo é necessário que os pacientes sejam vistos de modo integralizado e que a garantia à saúde possa alcançar até mesmo os locais periféricos (Pinto et al., 2021).

As mulheres negras são consideradas um grupo em situação de risco específico para a sífilis em gestantes, já que as evidências epidemiológicas apontam as tendências relativas a essa infecção como estacionárias ou crescentes na maioria das regiões brasileiras, com exceção da sífilis gestacional em mulheres pretas no Centro-Oeste e Sul, assim como no Brasil. Essa doença, responsável por altos índices de abortamento, natimortalidade, nascimento prematuro e que pode, ainda, ter como agravo a ocorrência da sífilis congênita, constitui um problema de saúde pública (de Morais et al., 2019), já que pode ser tanto evitada quanto tratada, especialmente, na Atenção Primária à Saúde (APS).

Entretanto, no âmbito desse nível de atenção também foram observados baixos resultados para negras em relação às brancas no que diz respeito à qualidade do acompanhamento pré-natal. Quando comparadas às mulheres brancas, as gestantes negras apresentam menor número de consultas e de exames durante o pré-natal - média de menos de 06 consultas para esse grupo, quando o recomendado pelos protocolos e diretrizes clínicas nacionais é a realização de, no mínimo, 07. Mulheres pardas e pretas 
menos escolarizadas, pertencentes às classes econômicas mais baixas, concentradas nas regiões Norte e Nordeste, são também as que recebem menos orientação durante o pré-natal sobre possíveis complicações na gravidez e início do trabalho de parto (Leal et al., 2017).

Dados do estudo Nascer Brasil revelam que nas regiões Sudeste e Sul do país, detentoras de população feminina branca em sua maioria, $83 \%$ das mulheres realizam 06 ou mais consultas no pré-natal. Já no Norte e Nordeste, de mulheres majoritariamente negras, esse número cai para 57\% e 67\%, respectivamente, com maior proporção de gestantes que declararam ter realizado três consultas ou menos nessas regiões (da Silva Nunes et al., 2017). Esses dados revelam a existência de possíveis lacunas no atendimento pré-natal prestado à mulher negra que podem contribuir para a manutenção das tendências crescentes da sífilis em gestantes pardas e pretas na maior parte das regiões brasileiras e, consequentemente, dos indicadores relativos à sífilis congênita, já que sabe-se que a prevenção desse agravo pode ser feita a partir da captação precoce da gestante, implantação da testagem rápida nas unidades primárias de saúde, diagnóstico da doença em tempo hábil e tratamento adequado da mulher e de suas parcerias sexuais (Oliveira Guanabara et al., 2017).

Outros fatores que comprometem a qualidade do cuidado pré-natal prestado a essas mulheres e que impactam na ocorrência de sífilis são a falta de interação entre o profissional de saúde e a gestante, comprometendo o acolhimento e o vínculo entre esses dois agentes, a falta de escuta e aconselhamento às demandas que a gestante apresenta e a desconsideração das singularidades, subjetividades e realidade da vida daquela mulher que se apresenta ao serviço de saúde (Oliveira Guanabara et al., 2017).

De forma semelhante à sífilis adquirida e em gestantes, a sífilis congênita tem sido associada a fatores sociais, econômicos, de infraestrutura e de acesso aos serviços de saúde, acometendo, muitas vezes, populações em situação de maior vulnerabilidade social. Dessa forma, uma das explicações para a ausência de tendências decrescentes de sífilis congênita em mulheres pardas de todas as regiões do Brasil seria o aumento das iniquidades sociais (Heringer et al., 2006). Os problemas que fragilizam a prevenção dessa infecção estão intimamente relacionados à assistência pré-natal prestada a essas mulheres e são estes: ausência da realização e atraso na entrega dos exames; abandono de pré-natal; falta de captação e resgate das gestantes faltosas; dificuldade no manejo da infecção por parte dos profissionais; dificuldade na captação e tratamento das parcerias sexuais; realização do diagnóstico da sífilis na gestante apenas no momento do parto/curetagem, falta de seguimento das mães e crianças após o parto; além da presença de dados incompletos nos prontuários e fichas de notificação (Cardoso et al., 2018; Melo et al., 2011).

Nesse sentido, as diferenças existentes na qualidade da atenção voltada a esse público e as experiências relacionadas à discriminação racial podem trazer consequências permanentes para indivíduos e coletividades, além de contribuir para a adoção de comportamentos inadequados, baixa adesão ao tratamento e mesmo adoecimento, aumentando, assim, a possibilidade de ocorrência de casos de doenças infecciosas, como a sífilis congênita (Leal et al., 2017).

Quanto à presença de tendências decrescentes de ocorrência de sífilis congênita em mulheres pretas, observa-se uma lacuna nos registros do item raça/cor nos instrumentos de notificação. Isso ocorre pela dificuldade na compreensão do que é "ser negro", "raça", "cor", impossibilitando um correto preenchimento dessa variável nos sistemas de informação em saúde. Outro agravo se constitui no fato de que o dado raça/cor é algo relativamente atual nos registros de saúde no Brasil e nem sempre há captação dos responsáveis pelo preenchimento desse campo (Matos \& Tourinho, 2018). Isso implica prejuízo na mensuração da atuação do Sistema Único de Saúde (SUS) para esse grupo populacional, além de dificultar estratégias de atenuação dos problemas que permeiam a população negra (Braz, 2013).

É difícil categorizar a raça/cor no Brasil, principalmente diante do colorismo brasileiro, fruto da miscigenação, responsável por gerar diferentes tons de pele, sendo a mais retinta, a mais marginalizada. Grande parte dessa miscigenação vem da tentativa de embranquecimento da população para atenuar o fenótipo negro no Brasil (Silva e Silva, 2017). A identidade negra 
foi estereotipada e dissociada, a exemplo do desconhecimento sobre algumas contribuições africanas no cotidiano brasileiro. Dessa forma, há uma desvalorização da ancestralidade negra (Dourado, 2019).

Essa ausência de reconhecimento expressa-se nos dados colhidos pelo IBGE que estão em coadunação com esse estudo, visto que a tendência da sífilis congênita cresce em pardas e decresce em negras em todas as regiões do Brasil. Diante deles, pode-se inferir que o dado está acompanhado por um processo de desvalorização da história negra. Dessa forma, tal dado pode estar, então, subnotificado para o campo raça/cor (Petruccelli \& Saboia, 2013). No entanto, apesar da existência dessa problemática, nota-se, ao longo dos anos, um entendimento cada vez maior sobre a temática e verifica-se a presença de uma parcela cada vez mais significativa da população brasileira que se autodeclara preta em consequência da atuação crescente do movimento negro brasileiro nos aspectos político, cultural e ideológico (Dourado, 2019).

A identificação racial - destituída de discriminação - é indispensável nos serviços de saúde tanto para auxiliar no diagnóstico, prognóstico e prevenção quanto para acompanhamento condigno do usuário, sobretudo das doenças consideradas raciais, pois permite a delimitação precisa do descaso, da omissão e da dificuldade de acesso, assim como possibilita perceber a institucionalização do racismo como prática social e políticas naturais e aceitáveis (Cordeiro, 2006), quando qualquer um desses grupos vive sob opressão racial.

\section{Considerações Finais}

A população negra se configura como importante parcela usuária dos SUS, no entanto, há dificuldade em contemplar a raça negra integralmente devido à presença do racismo institucional e o impacto histórico que ele proporciona.

Ao analisar a sífilis como um grave problema de saúde pública que atinge continuamente as mulheres negras nas diferentes regiões geográficas do Brasil, torna-se evidente a necessidade de políticas direcionadas para esse grupo.

Considerando as três formas de manifestação da infecção (adquirida, gestacional e congênita), é imprescindível o planejamento e o desenvolvimento de ações de educação em saúde, o rastreamento da infecção na população negra sexualmente ativa, diagnóstico precoce, bem como a realização da testagem para sífilis no pré-natal e tratamento adequado para a gestante e sua parceria sexual, conforme preconizado pelo Ministério da Saúde, a fim de se evitar a ocorrência de sífilis congênita. Esse conjunto de medidas contribui para atenuação dos indicadores de sífilis na população negra.

Entretanto, essa diminuição da ocorrência de sífilis na população negra só se torna totalmente alcançável quando se conhece as vulnerabilidades, as desigualdades socioeconômicas, culturais e as dificuldades de acesso à saúde enfrentadas por essa parcela da população brasileira. Por isso, é fundamental que haja o preenchimento adequado do campo raça/cor nos sistemas de informação em saúde para que se consiga tal entendimento da problemática que envolve o negro e, consequentemente, os avanços na atenção à saúde. $\mathrm{O}$ adequado preenchimento pode contribuir para o desenvolvimento de pesquisas que relacionem a sífilis ao recorte racial, criando a base para que se possa fundamentar ações públicas de enfrentamento desse quadro assistencial que atingem a população negra, visando a diminuição das iniquidades sociais geradas no âmbito da saúde e do SUS.

Diante desse cenário, este artigo recomenda aos trabalhos futuros a busca do conhecimento e compreensão das vulnerabilidades às quais estão expostas as mulheres negras no âmbito da saúde, apontando também para a necessidade de criação e manutenção de ações intervencionistas direcionadas a essa população, com o propósito de atenuar os indicadores relativos à sífilis e outras infecções sexualmente transmissíveis nas mulheres negras.

\section{Referências}

Almeida Filho, N. D., \& Barreto, M. L. (2011). Epidemiologia \& saúde: fundamentos, métodos, aplicações. $165-174$.

Avelleira, J. C. R., \& Bottino, G. (2006). Sífilis: diagnóstico, tratamento e controle. Anais brasileiros de dermatologia, 81, 111-126. https://www.scielo.br/j/abd/a/tSqK6nzB8v5zJjSQCfWSkPL/?lang=pt. 
Batista, L. E., Monteiro, R. B., \& Medeiros, R. A. (2013). Iniquidades raciais e saúde: o ciclo da política de saúde da população negra. Saúde em Debate, 37, 681-690. https://www.scielo.br/j/sdeb/a/spQ7FXCVNsJsKyHn8JzWMvj/?format=pdf\&lang=pt.

Brasil, Ministério da Saúde. Departamento de Doenças Crônicas e Infecções Sexualmente Transmissíveis. http://www.aids.gov.br/pt-br/publico-geral/infeccoessexualmente-transmissiveis/sifilis.

Brasil. Ministério da Saúde (2020). Secretaria de Vigilância em Saúde. Boletim Epidemiológico Sífilis 2020. Brasília. http://www.aids.gov.br/ptbr/pub/2020/boletim-sifilis-2020.

Braz, R. M., Oliveira, P. D. T. R. D., Reis, A. T. D., \& Machado, N. M. D. S. (2013). Avaliação da completude da variável raça/cor nos sistemas nacionais de informação em saúde para aferição da equidade étnico-racial em indicadores usados pelo Índice de Desempenho do Sistema Único de Saúde. Saúde em Debate, 37, 554-562. https://www.scielo.br/j/sdeb/a/ZqDr6yqgFryL5zXqCyrLVLc/?lang=pt\&format=html.

Cardoso, A. R. P., Araújo, M. A. L., Cavalcante, M. D. S., Frota, M. A., \& Melo, S. P. D. (2018). Análise dos casos de sífilis gestacional e congênita nos anos de 2008 a 2010 em Fortaleza, Ceará, Brasil. Ciência \& Saúde Coletiva, 23, 563-574. https://www.scielo.br/j/csc/a/Vj48x4jCTfP3jsRvgwrbBfd/?lang=pt.

Cordeiro, A. M. N. R. (2006). Saúde da População negra: Um espaço de ausências. Padê: Estudos em filosofia, raça, gênero e direitos humanos, 1(1). https://www.arqcom.uniceub.br/pade/article/view/133.

Crenshaw, K. (1989). Demarginalizing the intersection of race and sex: A black feminist critique of antidiscrimination doctrine, feminist theory and antiracist politics. u. Chi. Legal f., 139. https://heinonline.org/HOL/LandingPage?handle=hein.journals/uchclf1989\&div=10\&id=\&page= .

da Cruz Santos, T. (2019). As consequências da escravidão na história do negro no brasil. Diamantina Presença, 2(1), 47-57. https://www.revistas.uneb.br/index.php/diamantina/article/view/7381.

da Silva Nunes, A. D., Amador, A. E., Dantas, A. P. D. Q. M., de Azevedo, U. N., \& Barbosa, I. R. (2017). Acesso à assistência pré-natal no Brasil: análise dos dados da Pesquisa Nacional de Saúde. Revista Brasileira em Promoção da Saúde, 30(3). https://periodicos.unifor.br/RBPS/article/view/6158.

de Morais, T. R., Feitosa, P. W. G., de Oliveira, I. C., Girão, M. M. F., da Silva Sales, W., Brito, E. A. S., \& de Sousa Tavares, W. G. (2019). Interseccionalidades em Saúde: Predomínio de Sífilis Gestacional em Mulheres Negras e Pardas no Brasil. Revista de psicologia, 13(45), 670-679. https://idonline.emnuvens.com.br/id/article/view/1772.

de Paiva Terra, B., Krupp da Luz, C., \& Ferreira Resende, A. (2020). Machismo ou racismo? A interseccionalidade como resposta à hierarquia de opressões. Esferas, 18. https://portalrevistas.ucb.br/index.php/esf/article/view/11933.

Dourado, A. D. L. F., Araújo, L. I. L., \& Mori, V. D. (2019). Reflexões sobre o que é o "ser preto": os desafios e dilemas do reconhecimento de uma identificação racial num país miscigenado. Programa de Iniciação Científica-PIC/UniCEUB-Relatórios de Pesquisa.https://www.gti.uniceub.br/pic/article/view/7515.

Heringer, A. L. D. S., Kawa, H., Fonseca, S. C., Brignol, S. M. S., Zarpellon, L. A., \& Reis, A. C. (2020). Desigualdades na tendência da sífilis congênita no município de Niterói, Brasil, 2007 a 2016. Revista Panamericana de Salud Pública, 44 (8). https://www.scielosp.org/article/rpsp/2020.v44/e8/pt/.

Instituto Brasileiro de Geografia e Estatística (2019). Agência de Notícias. Pesquisa Nacional por Amostra de Domicílios Contínua. https://agenciadenoticias.ibge.gov.br/media/com_mediaibge/arquivos/8ff41004968ad36306430c82eece3173.pdf.

Leal, M. D. C., Gama, S. G. N. D., Pereira, A. P. E., Pacheco, V. E., Carmo, C. N. D., \& Santos, R. V. (2017). A cor da dor: iniquidades raciais na atenção prénatal e ao parto no Brasil. Cadernos de Saúde Pública, 33. https://www.scielo.br/j/csp/a/LybHbcHxdFbYsb6BDSQHb7H/abstract/?lang=pt\&format=html.

Lopes, F. (2005). Experiências desiguais ao nascer, viver, adoecer e morrer: tópicos em saúde da população negra no Brasil. Em Seminário saúde da população negra Estado de São Paulo 2004 (pp. 53-101).

Macêdo, V. C. D., Lira, P. I. C. D., Frias, P. G. D., Romaguera, L. M. D., Caires, S. D. F. F., \& Ximenes, R. A. D. A. (2017). Fatores de risco para sífilis em mulheres: estudo caso-controle. Revista de Saúde Pública, 51. https://www.scielo.br/j/rsp/a/NQhm4fVf7cqDnvDMGQpmGsD/?lang=pt.

Mariosa, D. F. (2019). Florestan Fernandes e os aspectos socio-históricos de uma integração híbrida no Brasil. Sociologias, 21, 182-209. https://seer.ufrgs.br/sociologias/article/view/81516.

Matos, C. C. D. S. A., \& Tourinho, F. S. V. (2018). Saúde da População Negra: percepção de residentes e preceptores de Saúde da Família e Medicina de Família e Comunidade. Revista Brasileira de Medicina de Família e Comunidade, 13(40), 1-12. https://rbmfc.org.br/rbmfc/article/view/1706.

Melo, N. G. D. O., Melo Filho, D. A. D., \& Ferreira, L. O. C. (2011). Diferenciais intraurbanos de sífilis congênita no Recife, Pernambuco, Brasil (20042006). Epidemiologia e Serviços de Saúde, 20(2), 213-222. http://scielo.iec.gov.br/scielo.php?pid=S167949742011000200010\&script=sci_abstract.

Oliveira Guanabara, M. A., Leite-Araújo, M. A., Matsue, R. Y., Lima de Barros, V., \& Alves Oliveira, F. (2017). Acesso de gestantes às tecnologias para prevenção e controle da sífilis congênita em Fortaleza-Ceará, Brasil. Revista de Salud Pública, 19, 73-78. https://www.scielosp.org/article/rsap/2017.v19n1/73$78 /$.

Petruccelli, J. L., \& Saboia, A. L. (Eds.). (2013). Características étnico-raciais da população: classificações e identidade. Instituto Brasileiro de Geografia e Estatística.

Pinto, L. F. D. S., Perini, F. D. B., Aragón, M. G., Freitas, M. A., \& Miranda, A. E. (2021). Protocolo Brasileiro para Infecções Sexualmente Transmissíveis 2020: infecção pelo HIV em adolescentes e adultos. Epidemiologia e Serviços de Saúde, 30. https://www.scielo.br/j/ress/a/N3PFzwZKhgLVPHngzGRFdfy/. 
Research, Society and Development, v. 11, n. 1, e51511125202, 2022

(CC BY 4.0) | ISSN 2525-3409 | DOI: http://dx.doi.org/10.33448/rsd-v11i1.25202

Santos, I. A. D. S (2015). O peso da cor no acesso aos direitos fundamentais. Curitiba: Universidade Federal do Paraná, 42. https://acervodigital.ufpr.br/bitstream/handle/1884/52688/R\%20-\%20E\%20-\%20IVANETE\%20APARECIDA\%20DA\%20SILVA\%20SANTOS.pdf?sequenc $\mathrm{e}=1 \&$ is Allowed $=\mathrm{y}$.

Santos, N. J. S. (2016). Mulher e negra: dupla vulnerabilidade às DST/HIV/aids. Saúde $e$ Sociedade, 25, 602-618. https://www.scielo.br/j/sausoc/a/B8LmxH9RGg3mbSm34SGSBDD/?lang=pt.

Silva e Silva, T. (2017). O colorismo e suas bases históricas discriminatórias. Direito UNIFACS-Debate Virtual, (201). https://revistas.unifacs.br/index.php/redu/article/view/4760.

Souzas, R., Marinho, O. F. P., \& Melo, K. L. O. (2012). Acesso à saúde, promoção e prevenção ao HIV/AIDS e o recorte racial/étnico: revisão bibliográfica (1995-2009). Batista LE, Werneck J, Lopes F, orgs. Saúde da população negra. Petrópolis: DP et Alii, 266-86.

Werneck, J. (2016). Racismo institucional e saúde da população negra. Saúde e Sociedade, 25, 535-549. https://www.scielo.br/j/sausoc/a/bJdS7R46GV7PB3wV54qW7vm/?lang=pt. 